\title{
Lyotropic Liquid Crystalline Mesophase Governs Interfacial Molecular Orientation of Conjugated Polymer Thin Films
}

Ge Qu,${ }^{\dagger}$ Kyungsun Park, ${ }^{\dagger}$ Prapti Kafle,,$^{\dagger}$ Fengjiao Zhang, ${ }^{\dagger}$ Justin J. Kwok, ${ }^{\S}$ Bijal Patel, ${ }^{\dagger}$ DetlefM. Smilgies," Lars Thomsen, ${ }^{+\dagger}$ Christopher R. McNeill, $\neq \neq$ Ying Diao ${ }^{*}{ }^{\dagger}$

†Department of Chemical and Biomolecular Engineering, University of Illinois Urbana-Champaign, 600 S. Mathews Avenue, Urbana, Illinois 61801, United States

‡School of Chemical Sciences, University of Chinese Academy of Science, Beijing 100049, China

$\S$ Department of Materials Science and Engineering, University of Illinois at UrbanaChampaign,1304 W. Green St., Urbana, IL 61801, USA

"Cornell High Energy Synchrotron Source (CHESS), Ithaca, New York 14853, United States ${ }^{\dagger+}$ The Australian Synchrotron, 800 Blackburn Road, Clayton, Victoria 3168, Australia ‡ÆDepartment of Materials Engineering, Monash University, Clayton, Victoria 3800, Australia Corresponding author: yingdiao@illinois.edu 


\section{Supplementary Methods.}

Substrate treatment. Multiple surface treatments were performed to vary the substrate surface energy. Octyldecyltrichlorosilane (OTS) treatment was performed by immersing a plasma treated silicon wafer with $300 \mathrm{~nm} \mathrm{SiO}$ layer in $50 \mathrm{~mL}$ of trichloroethylene mixed with $100 \mu \mathrm{L}$ OTS at room temperature for 20 minutes. The wafer was then washed with solvents and baked at $120{ }^{\circ} \mathrm{C}$ for 20 minutes to crystallize OTS. Poly(vinylidene fluoride-co-hexafluoropropylene) (PVDFHFP) substrate was prepared by spin-coating 1:8 weight ratio PVDF-HFP : acetone solution onto plasma treated $300 \mathrm{~nm} \mathrm{SiO} 2$ substrate at $3000 \mathrm{rpm}$ for 1 minute. The PVDF-HFP substrates was heated at $80^{\circ} \mathrm{C}$ in the glovebox overnight. Phenyltrichlorosilane (PTS) treatment was performed by heating the immersed wafer in $50 \mathrm{~mL}$ of toluene mixed with $1.5 \mathrm{~mL}$ PTS at $90{ }^{\circ} \mathrm{C}$ for $12-15$ hours. The wafer was rinsed with solvents and sonicated in toluene for 3 minutes before drying. Poly(4-vinylphenol) and 4,4'-(hexafluoroisopropylidene)diphthalic anhydride (PVP:HDA) substrate was prepared by spin coating PVP and HDA in propylene glycol methyl ether acetate (PEGMA) solution (20 mg/ml for PVP and $2 \mathrm{mg} / \mathrm{ml}$ for HDA) at $7000 \mathrm{rpm}$ for 1 minute. The PVP:HDA substrates were heated at $100{ }^{\circ} \mathrm{C}$ in glovebox for 1 hour and at $60{ }^{\circ} \mathrm{C}$ in vacuum oven for 1 hour.

SAXS measurements. Small angle X-ray scattering (SAXS) was performed at beamline 12-IDB at the Advanced Photon Source at the Argonne National Laboratory. Liquid samples were measured in $2.0 \mathrm{~mm}$ quartz capillary tubes and solid samples are measured in aluminum sample pans for dynamic scanning calorimetry. Data were collected on a two-dimensional Pilatus $2 \mathrm{M}$ detector with an X-ray beam energy at $13.3 \mathrm{keV}$. In situ thermal SAXS experiments were carried on a Linkam THMS600 stage from $25{ }^{\circ} \mathrm{C}$ to $125{ }^{\circ} \mathrm{C}$ for liquid samples and to $300{ }^{\circ} \mathrm{C}$ for solid samples. The collected data were azimuthally integrated into one-dimensional scattering profiles. Background correction was applied with respect to solution filled capillary or empty aluminum pan when necessary. 


\section{Supplementary Notes.}

Solution state SAXS results and discussion. We performed solution SAXS measurements (Figure S5) to understand the molecular ordering and structures of the LC mesophase of DPPBTz in chlorobenzene. The polymer solution was placed in a quartz capillary on a heating stage to run transmission SAXS experiments at various temperatures. In Figure S5a, A broad peak arises in the range of $\mathrm{q}=0.1-0.5 \AA^{-1}$ in the background subtracted SAXS 1D profile for both $10 \mathrm{mg} / \mathrm{ml}$ and $100 \mathrm{mg} / \mathrm{ml}$ DPP-BTz solution. The broad peak centers at $0.191 \AA^{-1}$, corresponding to a dspacing of $32.9 \AA$ assuming the predominant contribution from the structure factor. Given that this peak lies in the range of the solid state lamellar stacking peak, we performed transmission SAXS measurements to directly compare the two. Specifically, we packed DPP-BTz solid powder in a press-sealed thermogravimetric analysis (TGA) aluminum pan and performed SAXS scans at 25 ${ }^{0} \mathrm{C}$ and $300{ }^{0} \mathrm{C}$ (Figure S5c). The (100) lamellar stacking peak is much sharper, centering at $\mathrm{q}=$ $0.219 \AA^{-1}$ which is insensitive to temperature. The lamellar stacking peak distance is calculated to be $28.7 \AA$, agreeing with literature values. ${ }^{1,2}$ Given the close proximity with the (100) lamellar stacking peak (Figure S5d), we attribute the origin of the $0.191 \AA^{-1}$ peak DPP-BTz solution to loose lamella stacking, which appears in both low concentration solution state and the LC mesophase. Further, the rising intensity at the low q region indicates presence of pre-aggregates for both 10 and $100 \mathrm{mg} / \mathrm{ml}$ solutions. We infer that the loose lamella stacking occurs inside the preaggregates. Therefore, we infer that as the solution concentration increases, lamella stacking intensifies inside the polymer aggregates, while a high volume density of rigidified polymer aggregates further associate to form a "colloidal" liquid crystal phase. During film deposition, the loose lamella stacking in the LC phase at a spacing of $32.9 \AA$ evolves into close lamella stacking at a spacing of $28.7 \AA$. At the same time, the peak sharpens as the paracrystalline disorder drastically reduces from the LC phase to the solid state.

Effect of substrate surface free energy on out-of-plane orientation. Having understood the appearance of face-on LC mesophase near the substrate interface and in the bulk liquid layer, we look into the influence of substrate interaction with the conjugated polymer on the templating effect of the LC mesophase and the final orientation of crystallites in the film. We prepare a series of substrates with various substrate surface energy: OTS $(\gamma=20.5 \mathrm{mN} / \mathrm{m})$, PVDF-HFP $(22.4$ $\mathrm{mN} / \mathrm{m})$, PTS $(36.0 \mathrm{mN} / \mathrm{m})$, PVP:HDA $(41.7 \mathrm{mN} / \mathrm{m})$ and $\mathrm{SiO}_{2}(52.2 \mathrm{mN} / \mathrm{m})$, with the molecular structure of the substrate surface shown in Figure S7a. The surface energies of OTS, PTS, 
PVP:HDA and $\mathrm{SiO}_{2}$ were determined in previous work, and the surface energy of PVDF-HFP is calculated from its contact angles with probing liquids of known surface energies using the equation of state introduced in the same work. ${ }^{3}$ DPP-BTz thin films were deposited onto the substrates at $10 \mathrm{mg} / \mathrm{ml}$ concentration in chlorobenzene with $0.5 \mathrm{~mm} / \mathrm{s}$ coating speed at $80{ }^{\circ} \mathrm{C}$ (Figure S8). We carried out GIWAXS measurements for the top interface and bulk film and construct the corresponding partial pole figures for films coated on various substrates (Figure S7 $\mathbf{b}, \mathbf{c})$. The top interface from most substrates again shows the existence of edge-on crystallites, compared to the primarily face-on bulk film and bottom interface. However, the out-of-plane orientation does not show significant dependence on substrate surface energy, reflected in the calculated out-of-plane 2D orientation parameter (Figure S7d, tabulated in Table S2). The calculated 2D orientation parameter values concentrate near -0.4 , without significant interface/bulk film differentiation. The out-of-plane molecular orientation of the DPP-BTz substrate series is very similar to the previous speed series on $\mathrm{SiO}_{2}$, with edge-on crystallites at the top surface and face-on crystallites in the bulk. Hence, varying molecular interactions between polymer and substrate by using different modification layers does not change the out-of-plane orientation of DPP-BTz, validating that the solid state orientation is determined by formation of twisted LC mesophase in the solution state.

In the above characterization, we have shown that varying the molecular interaction between polymer and solid substrate does not alter the out-of-plane molecular orientation of DPP-BTz. On the other hand, our earlier work has demonstrated that by varying the amount of ionic liquid in the ion gel substrates, the orientation in DPP-BTz thin films shifts from face-on to edge-on with increasing amount of ionic liquid up to an optimum percentage. ${ }^{4}$ Ionic liquid is a dynamic template that significantly expedite polymer crystallization, leading to edge-on molecular orientation in the final film. Therefore the orienration control mechanism is different in the ealier work compared to this work, causing no contradiction with each other.. 
Supplementary Figures and Tables.

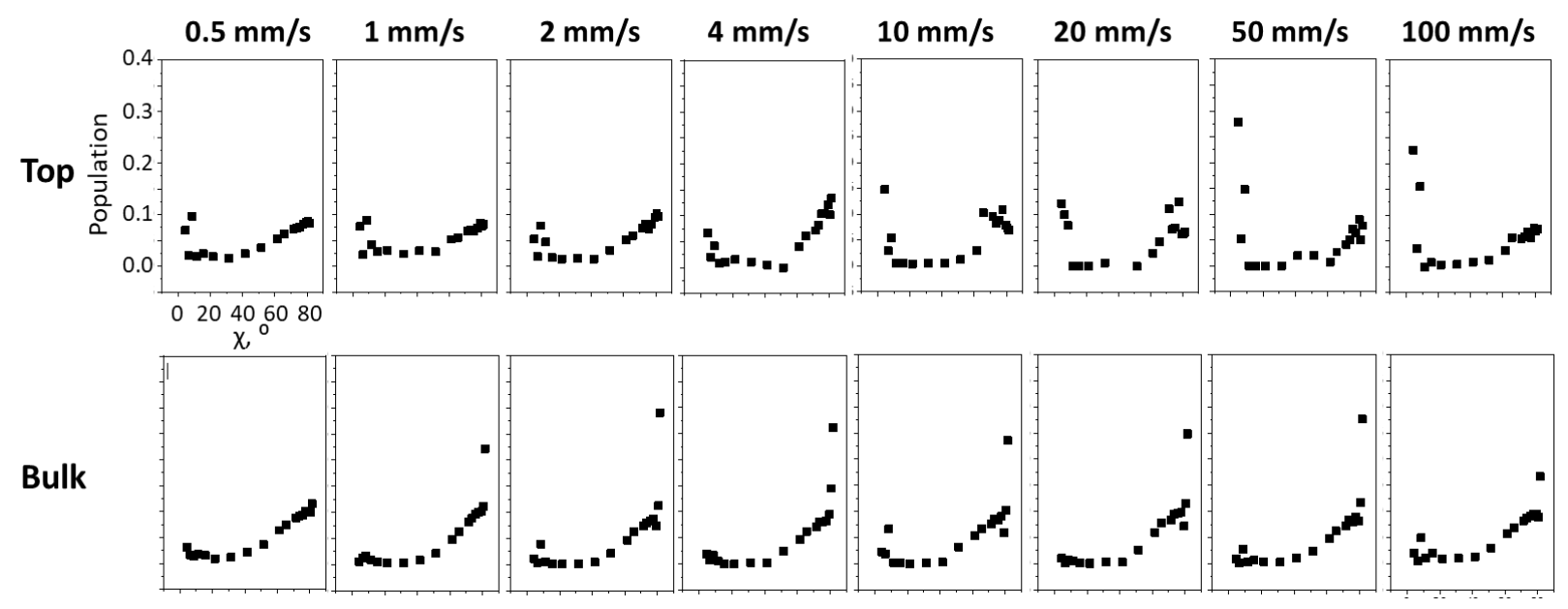

Figure S1. Partial pole figures for top and bulk measurements of DPP-BTz thin films coated on $\mathrm{SiO}_{2}$ substrates between $0.5 \mathrm{~mm} / \mathrm{s}$ and $100 \mathrm{~mm} / \mathrm{s}$ 


\section{(a)}

(b)
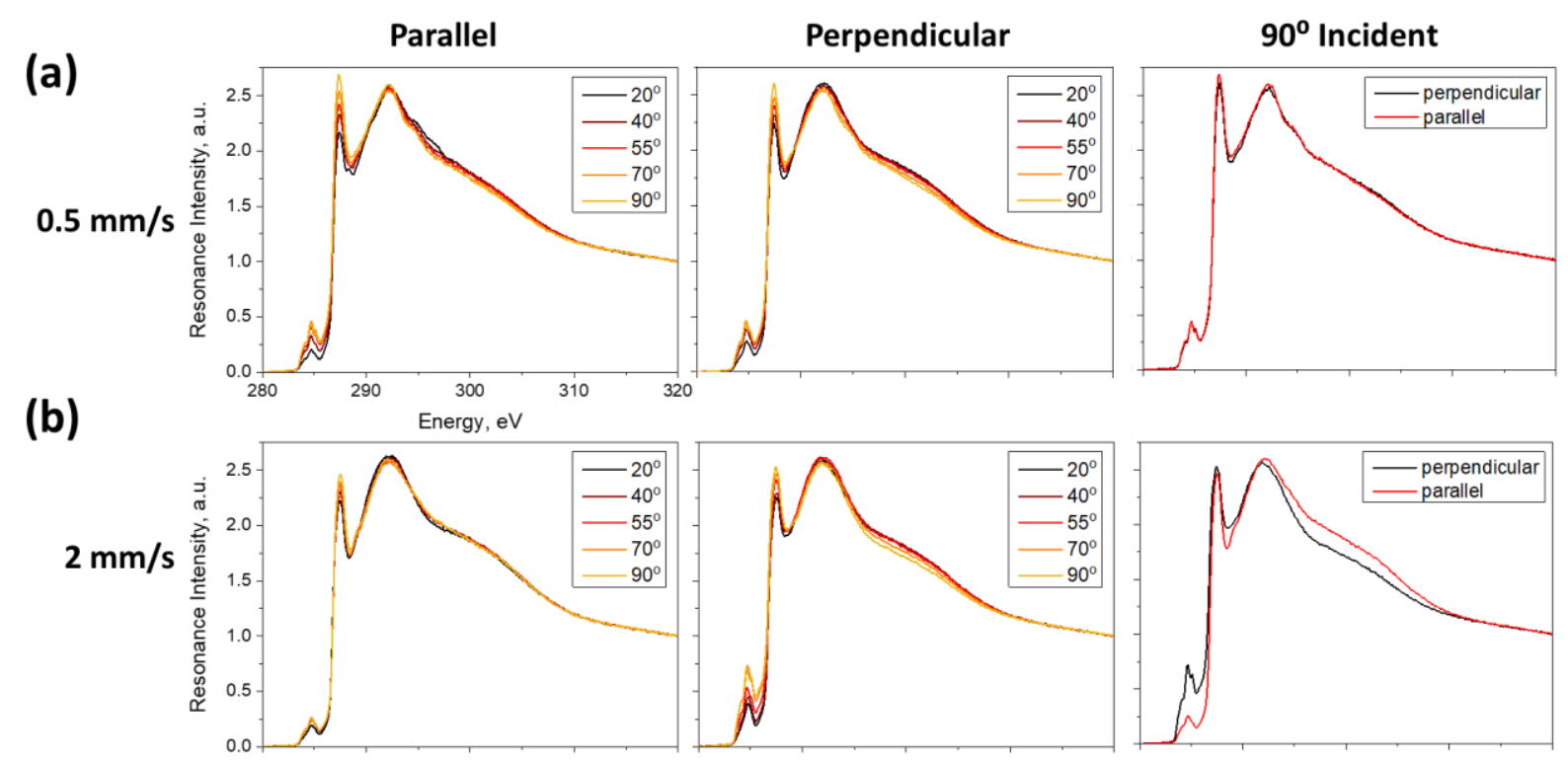

(c)
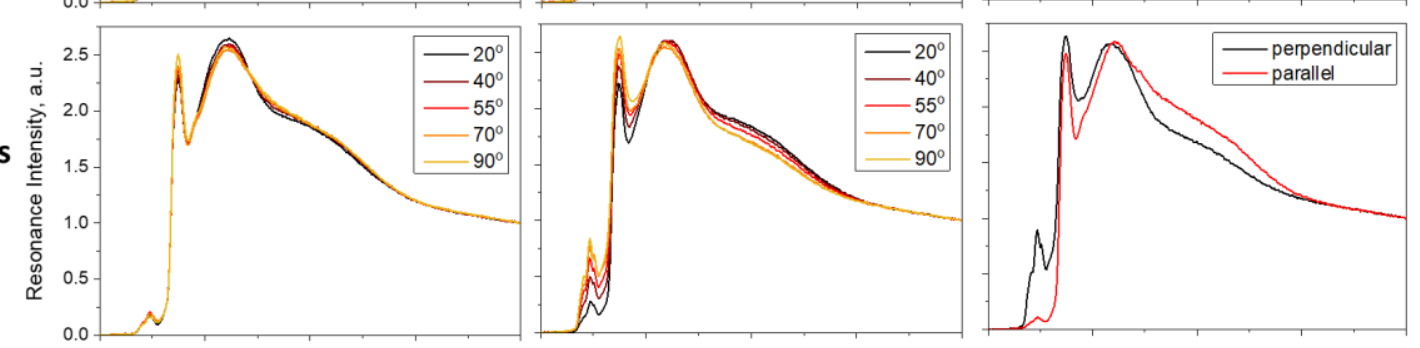

(d)
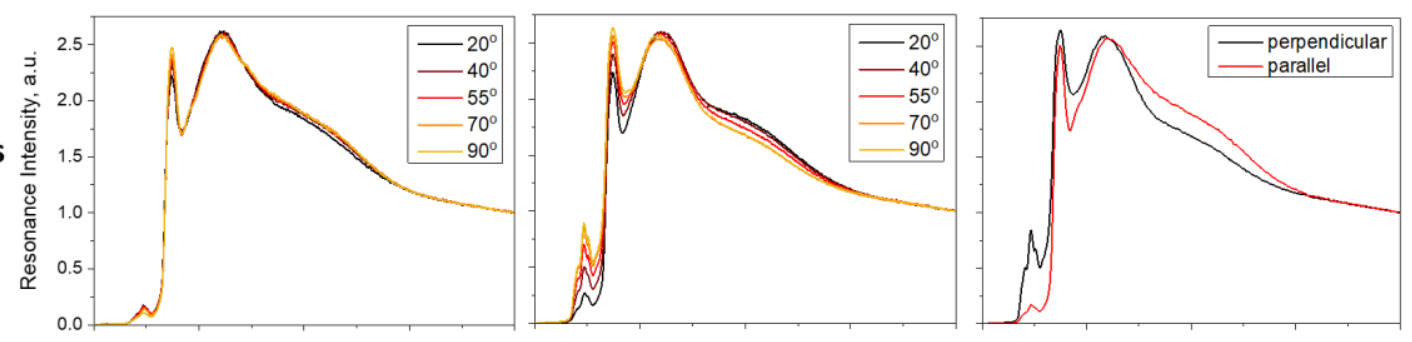

Figure S2. NEXAFS PEY tilt angle spectra of DPP-BTz thin films coated at (a) $0.5 \mathrm{~mm} / \mathrm{s}$, (b) 2 $\mathrm{mm} / \mathrm{s}$, (c) $4 \mathrm{~mm} / \mathrm{s}$ and (d) $100 \mathrm{~mm} / \mathrm{s}$, measured with the incident X-ray beam parallel and perpendicular to the coating direction. The parallel and perpendicular scans with $90^{\circ}$ incident angle are plotted for in-plane alignment evaluation. 
Table S1. Out-of-plane 2D orientation parameter of DPP-BTz speed series thin films from GIWAXS and NEXAFS measurements.

\begin{tabular}{|c|c|c|c|}
\hline $\begin{array}{c}\text { Coating speed, } \\
\mathrm{mm} / \mathrm{s}\end{array}$ & GIWAXS top & GIWAXS bulk & NEXAFS top \\
\hline 0.5 & -0.19 & -0.41 & 0.53 \\
\hline 1 & -0.09 & -0.58 & \\
\hline 2 & -0.23 & -0.60 & 0.61 \\
\hline 4 & -0.56 & -0.63 & 0.65 \\
\hline 10 & -0.39 & -0.51 & \\
\hline 20 & -0.21 & -0.65 & \\
\hline 50 & 0.07 & -0.56 & \\
\hline 100 & -0.03 & -0.41 & 0.68 \\
\hline
\end{tabular}




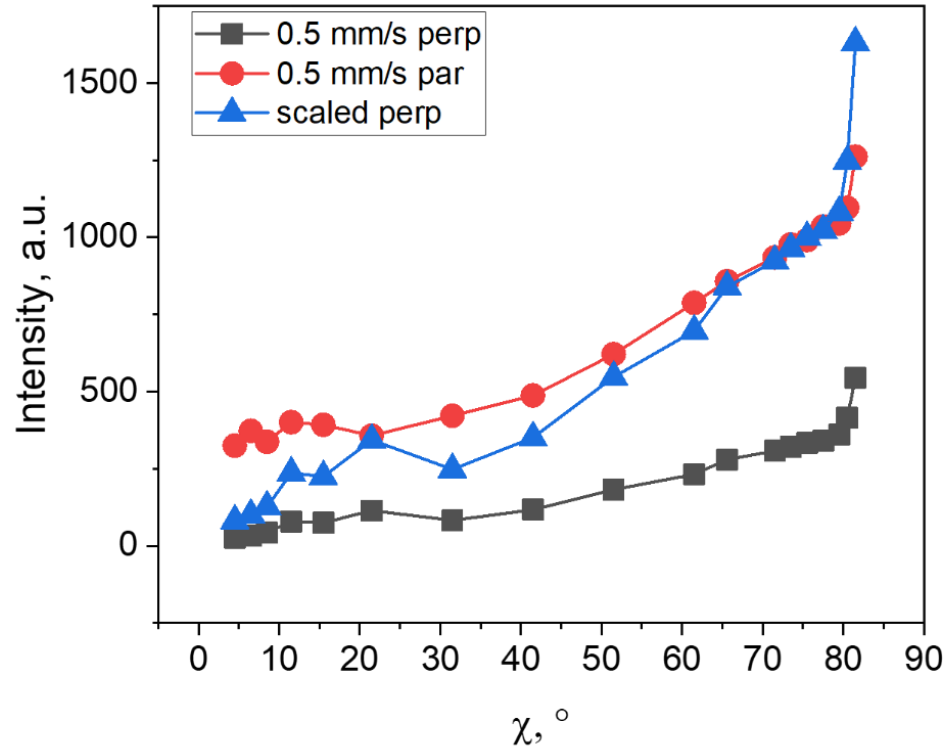

Figure S3. Pole figure comparison from DPP-BTz thin film coated from in situ $\mu$ GIWAXS measurements. Measurements is performed with the incident X-ray beam paralle and perpendicular to coating direction for films coated from $5 \mathrm{mg} / \mathrm{ml}$ DPP-BTz in chlorobenzene solution on $\mathrm{SiO}_{2}$ substrate at $70{ }^{\circ} \mathrm{C}$. The perpendicular intensity is scalled to compare with parallel intensity. 

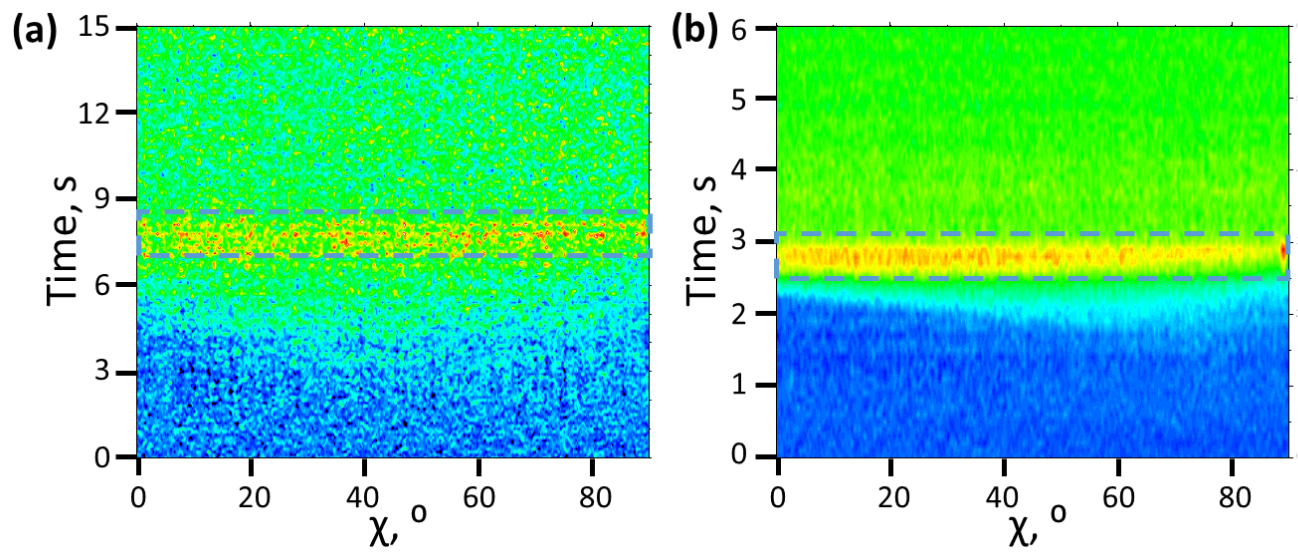

Figure S4. Normalized intensity-time- $\chi$ plot of the transient peak $\left(\mathrm{q}=0.8-0.95 \AA^{-1}\right)$ for solution coating of (a) pure chlorobenzene and (b) $5 \mathrm{mg} / \mathrm{ml}$ DPP-BTz in chlorobenzene solution. The time scale reflected real time but with arbitrary start point. 

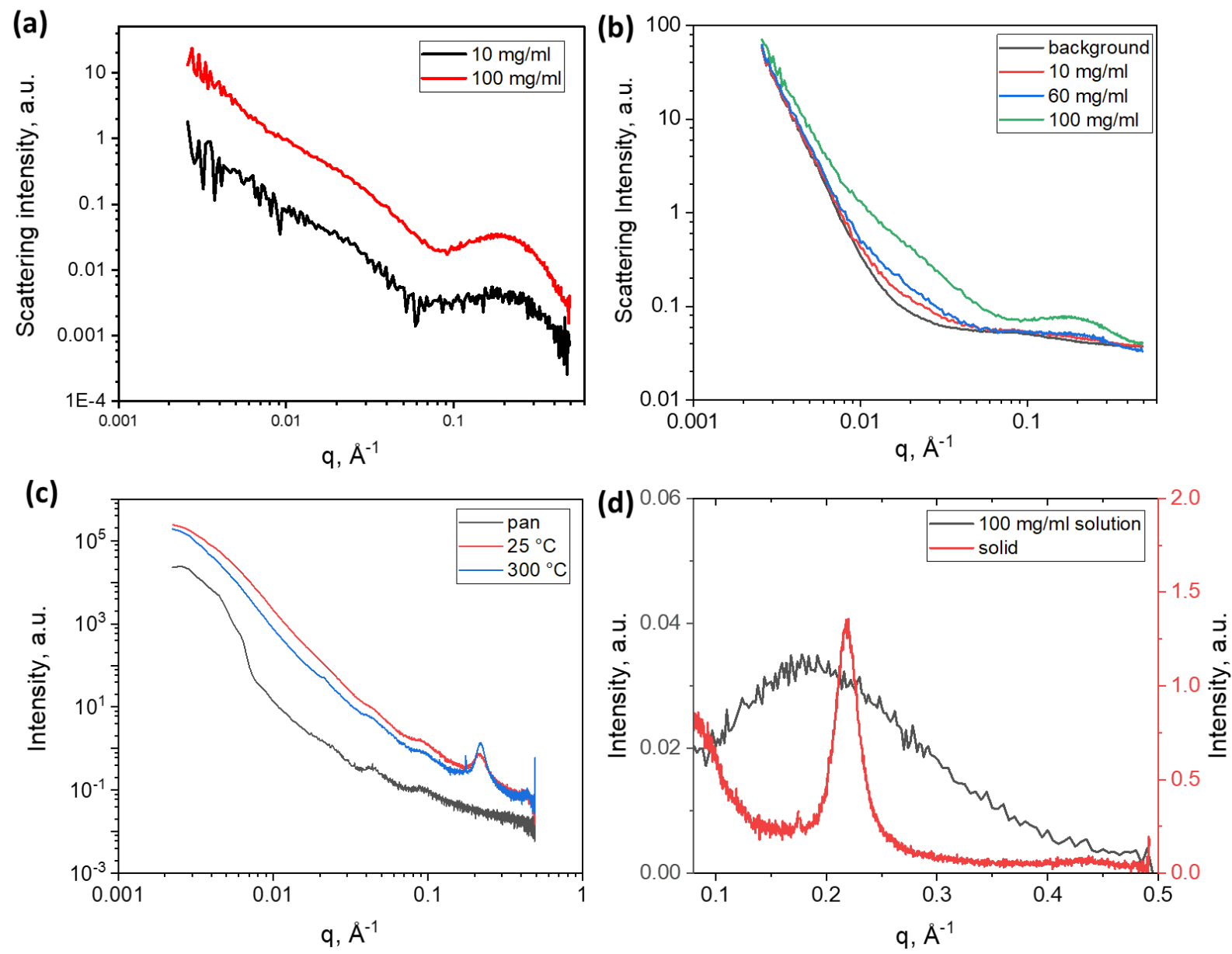

Figure S5. (a) Background subtracted SAXS 1D profile of $10 \mathrm{mg} / \mathrm{ml}$ and $100 \mathrm{mg} / \mathrm{ml} \mathrm{DPP-BTz} \mathrm{in}$ chlorobenzene solution at $85^{\circ} \mathrm{C}$. (b) Solution state SAXS 1D scattering profile of chlorobenzene background, $10 \mathrm{mg} / \mathrm{ml}, 60 \mathrm{mg} / \mathrm{ml}$ and $100 \mathrm{mg} / \mathrm{ml} \mathrm{DPP-BTz}$ in chlorobenzene solution at $85{ }^{\circ} \mathrm{C}$. (b) Solid state SAXS 1D scattering profile of aluminum pan background and DPP-BTz polymer solid at $25^{\circ} \mathrm{C}$ and $300{ }^{\circ} \mathrm{C}$. (d) Background subtracted SAXS 1D profile of $100 \mathrm{mg} / \mathrm{ml} \mathrm{DPP}-\mathrm{BTz}$ solution vs. DPP-BTz solid within the q range of 0.09 to $0.5 \AA^{-1}$, comparing the LC peak from solution with the (100) lamellar stacking peak of solid. 

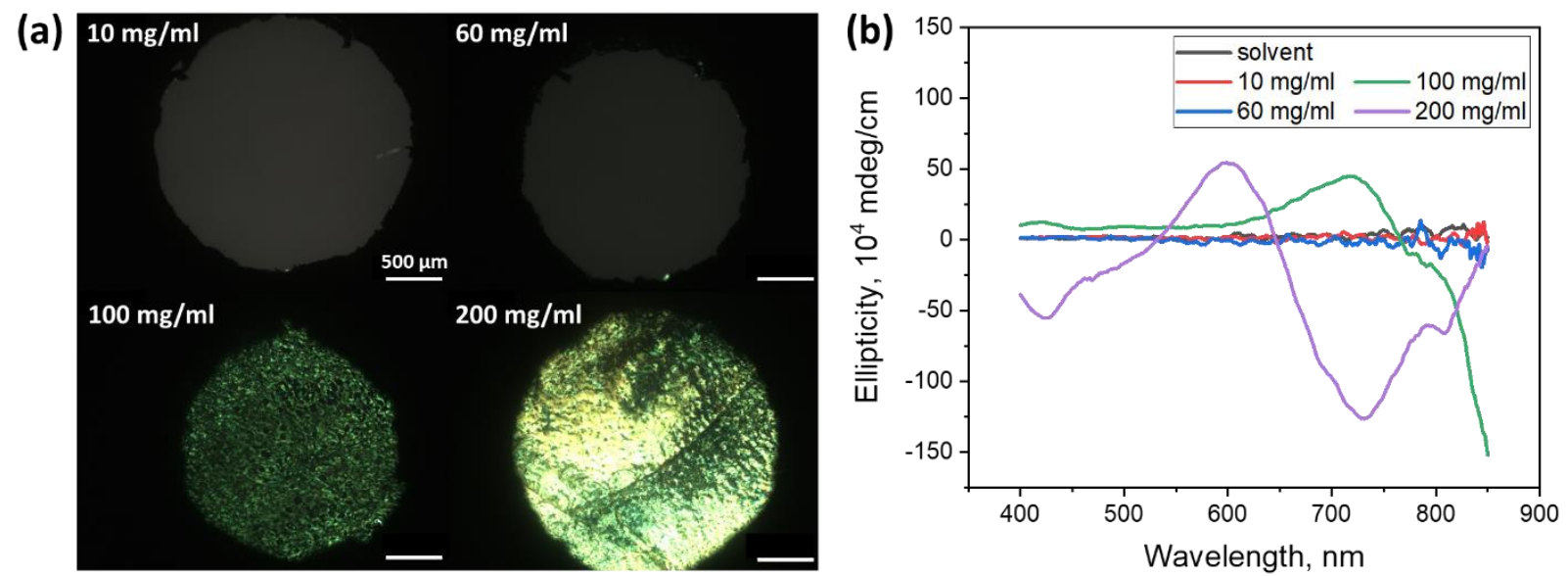

Figure S6. Solution state DPP-BTz (a) POM images and (b) corresponding CD spectra at various concentration. 
(a)

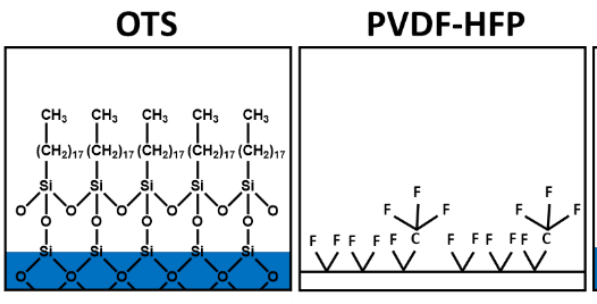

PTS

PVP:HDA

SiO2

(b)
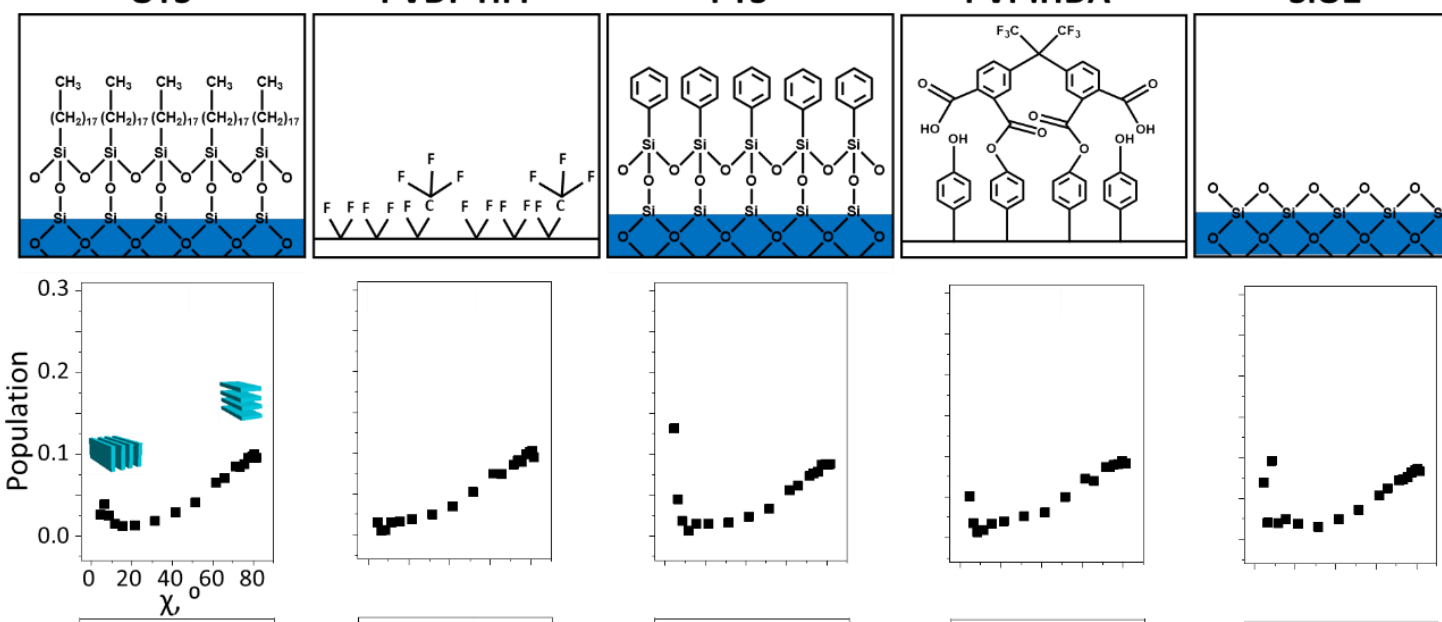

(c)
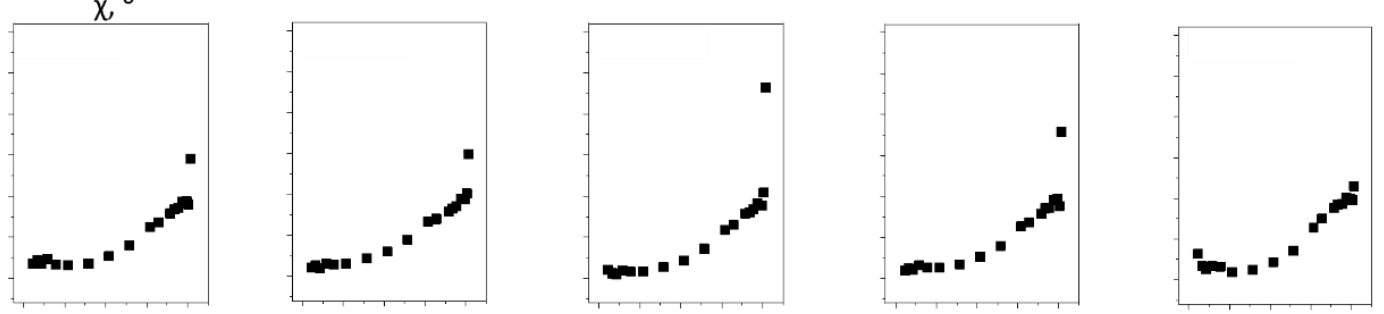

(d)

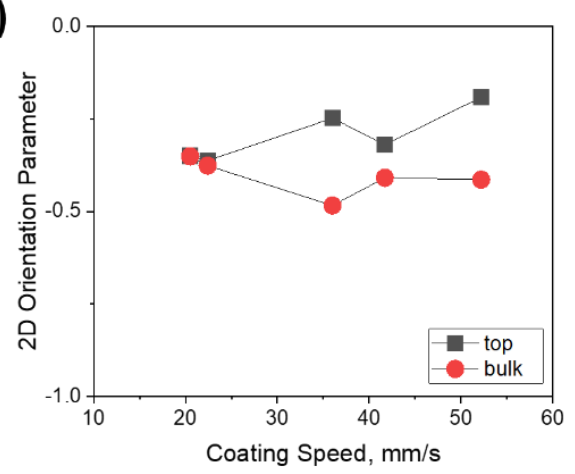

Figure S7. (a) Molecular structure of the substrate surface chemistry and partial pole figures of the (b) top interface and (c) bulk film of solution coated DPP-BTz on OTS, PVDF-HFP, PTS, PVP:HDA and $\mathrm{SiO} 2$ substrates at $0.5 \mathrm{~mm} / \mathrm{s}$ and $80{ }^{\circ} \mathrm{C}$. (d) Out-of-plane 2D orientation parameter from GIWAXS pole figures measurements for various substrates. 
OTS

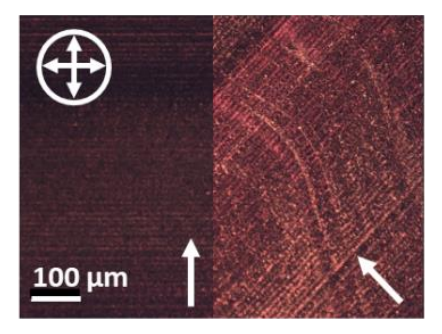

PVDF-HFP
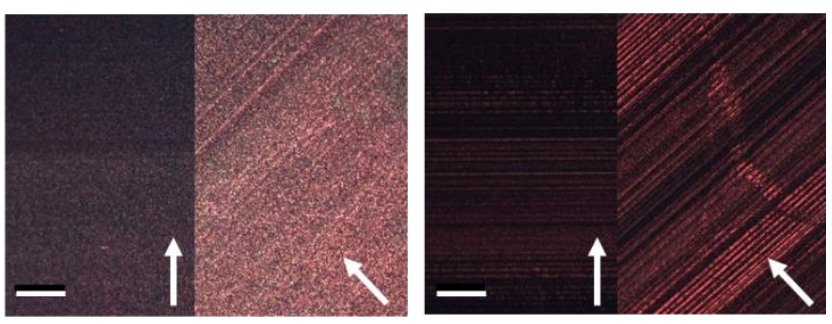

PVP:HDA

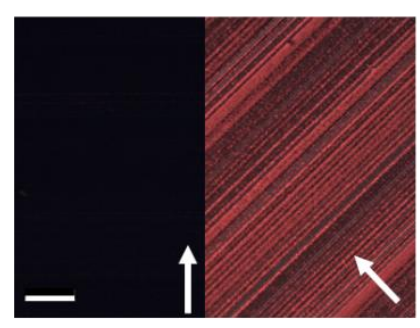

$\mathrm{SiO2}$

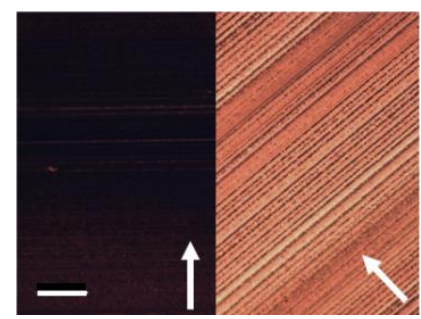

Figure S8. Cross polarized microscopy images of solution coated DPP-BTz on OTS, PVDF-HFP, PTS, PVP:HDA and $\mathrm{SiO}^{2}$ substrates at $0.5 \mathrm{~mm} / \mathrm{s}$ and $80^{\circ} \mathrm{C}$.

Table S2. Out-of-plane 2D orientation parameter of DPP-BTz substrate series thin films from GIWAXS measurements.

\begin{tabular}{|c|c|c|c|}
\hline Substrate & $\begin{array}{c}\text { Surface energy } \\
\mathrm{mN} / \mathrm{m}\end{array}$ & GIWAXS top & GIWAXS bulk \\
\hline OTS & 20.5 & -0.35 & -0.35 \\
\hline PVDF-HFP & 22.3 & -0.36 & -0.38 \\
\hline PTS & 36.0 & -0.25 & -0.48 \\
\hline PVP:HDA & 41.7 & -0.32 & -0.41 \\
\hline $\mathrm{SiO}_{2}$ & 52.2 & -0.19 & -0.41 \\
\hline
\end{tabular}




\section{References:}

1. Schott, S.; Gann, E.; Thomsen, L.; Jung, S. H.; Lee, J. K.; McNeill, C. R.; Sirringhaus, H., Charge-Transport Anisotropy in a Uniaxially Aligned Diketopyrrolopyrrole-Based Copolymer. Adv. Mater. 2015, 27, 7356-7364.

2. Mohammadi, E.; Qu, G.; Kafle, P.; Jung, S.-H.; Lee, J.-K.; Diao, Y., Design Rules for DynamicTemplate-Directed Crystallization of Conjugated Polymers. Molecular Systems Design \& Engineering 2020, 5, 125-138.

3. Zhang, F.; Mohammadi, E.; Luo, X.; Strzalka, J.; Mei, J.; Diao, Y., Critical Role of Surface Energy in Guiding Crystallization of Solution-Coated Conjugated Polymer Thin Films. Langmuir 2018, 34, 1109-1122.

4. Mohammadi, E.; Zhao, C.; Zhang, F.; Qu, G.; Jung, S.-H.; Zhao, Q.; Evans, C. M.; Lee, J.-K.; Shukla, D.; Diao, Y., Ion Gel Dynamic Templates for Large Modulation of Morphology and Charge Transport Properties of Solution-Coated Conjugated Polymer Thin Films. ACS Appl. Mater. Interfaces 2019, 11, 22561-22574. 